\title{
Phloem loading and unloading
}

\author{
S. Delrot and J.L. Bonnemain \\ Laboratoire de Physiologie et Biochimie Végétales, CNRS URA81, 25, rue du Faubourg Saint- \\ Cyprien, 86000 Poitiers, France
}

\section{Introduction}

Phloem transport of assimilates provides the materials needed for the build up of the herbaceous plant or the tree. Understanding this mechanism is therefore important to control the edification of the plant. Considerable work has been devoted to transport in the past (for recent reviews, see Giaquinta, 1983; Delrot and Bonnemain, 1985; Delrot, 1987, 1989; Van Bel, 1987), but much further work is needed, especially on woody species, because the information available on basic processes, such as loading into and unloading from the sieve tubes, mainly concerns herbaceous species. Therefore, this short overview will often refer to herbaceous species but the general principles which will be given may be used to understand assimilate transport in trees. Actually, the scant information available shows wide variety in the anatomical, physiological, and biochemical situations involved in assimilate transport.

\section{General background}

Nature of translocated substances

Long distance transport of assimilates occurs in specialized cells (sieve tubes) characterized by their osmotic pressure. The high osmotic pressure of the phloem sap is due to the presence of many solutes: sugars, amino acids, ions (Ziegler, 1975). Concerning sugars, in many species, sucrose is the predominant mobile sugar: This is the case for most herbaceous plants and for tree species belonging to gymnosperms (Picea abies, Pinus strobus) or angiosperms (monocotyledons, palm-tree; dicotyledons, willow). In other plants in addition to sucrose, the phloem sap contains oligosaccharides belonging to the raffinose family and characterized by the attachment of one or more galactose residues to the sucrose molecule. Some members of Bignoniaceae, Tiliaceae and Ulmaceae belong to this group of plants. A third group is made of species containing sugar alcohols in the phloem sap, for example mannitol (Oleaceae; Fraxinus, Syringa), sorbitol (Prunus serotina, Malus domestica), or dulcitol (Celastraceae). As regards amino acids, gluamine/glutamate and asparagine/aspartate are the quantitatively predominant compounds (1-30 mM each), together with serine, but there are exceptions. For example, proline is the predominant amino acid in the sieve tube sap, of Robinia. In some species, the phloem sap also contains ureides, allantoin and allantoic acid (Acer, Platanus, Aesculus) or citrul- 
line (Betula, Carpinus, Alnus, Juglans). There is no evidence that any of these nitrogenous substances is excluded from the sieve fubes, in contrast to the loading of sugars, which is a highly selective process. In all investigated cases, the predominant cation in sieve tube sap is potassium, while the predominant anion is generally phosphate and sometimes chloride. Another striking feature of the phloem sap is its alkaline $\mathrm{pH}$ (7.5-8.5). The concentration of the phloem sap exhibits nycthemeral variations (Hocking, 1980) and its content exhibits seasonal variations (Ziegler, 1975), as well as variations depending upon the location in the plant (Hocking, 1980; Vreugdenhil, 1985).

The different steps involved in long distance transport

Assimilate transport involves 3 steps which are lateral transport from the chloroplast to the conducting bundle in the leaf (source), translocation in the sieve tubes (path), and lateral transport from the sieve tubes to the receiving cells (sink). Lateral transport in the source, which ends in the active loading of the assimilates in the sieve tube, provides the driving force for translocation, while the activity in the different sinks controls the direction of transport. Although the presence of actin and myosin-like proteins in the phloem of some species may give support to the hypothesis of active translocation powered by contractile filaments (Kursanov et al., 1983; Turkina et al., 1987), translocation in the path is thought to be rather passive, particularly in species whose phloem transport is not sensitive to temperature for a wide range of values (Faucher et al., 1982). Yet, mechanisms must function in the stem to prevent excessive leakage of assimilates from the conducting tissue to the external parenchyma. In the following, attention will be paid mainly to the events occurring in the source and in the sink.

\section{Lateral transport and phloem loading in the leaf}

In the leaf, the assimilates which are not used for growth may be either stored in a storage compartment (vacuole or chloroplast) or exported via a mobile compartment (cytosol or endoplasmic reticulum). Lateral transport up to the conducting bundle may be apoplastic, in the cell wall, if assimilates are leaked into the apoplast, or symplastic, via the plasmodesmata which connect the mesophyll cells to one another. The final step of lateral transport is the active loading of assimilates into the conducting complex. Until recently, the only evidence available suggested that active loading occurred from the apoplast, but some authors now argue that loading might also occur via the plasmodesmata in some species.

Two markedly different examples will be given to illustrate the present status of knowledge, the diversity of the situations encountered, and the questions being debated.

\section{Apoplastic loading}

Evidence detailed elsewhere (Delrot, 1987, 1989, and references therein) shows that in Beta vulgaris and Vicia faba, loading of sugars is mediated by a proton-sucrose cotransport process across the plasmalemma of the conducting complex (companion cell-sieve tube). This evidence may be summarized as follows. Plasmolytic studies show the existence of a steep, uphill concentration gradient at the boundary of the sieve tube-companion cell complex. Loading is 
specific for sucrose, since exogenous hexoses are not absorbed by the veins. It is promoted by adenosine triphosphate, fusiccocin (an activator of the plasmalemma proton-pump), but inhibited by uncouplers and metabolic inhibitors. Sucrose is present in the apoplast and is the major mobile sugar. Apoplastic sucrose concentration undergoes nycthemeral changes and is sensitive to treatments which block export in various herbaceous species. The sieve tube is associated with specialized transfer cells possessing numerous wall ingrowths, which increase the volume of the apoplast and the surface area of plasmalemma available for exchanges. The sieve tube and the transfer cell are connected by plasmodesmata, but in contrast, very few plasmodesmata are found at the boundary between the conducting complex and the surrounding cells. In Vicia faba, the number of plasmodesmata decreases as the proximity of the cells considered to the conducting complex increases. The conducting complex is therefore an insulated unit, and all the properties described above strongly suggest apoplastic loading. The existence of a proton extruding activity more concentrated or more active in the veins than in the surrounding tissues, and the demonstration of sucrose-induced alkalizations of the medium indicate that uptake of sucrose in leaf tissues, and more particularly in the veins, occurs with proton cotransport. This is further substantiated by uptake experiments which show that the sucrose carrier obeys 2 substrate kinetics, with the proton and sucrose as the substrates. The sucrose carrier is able to recognize sucrose, maltose, raffinose and $\alpha$-phenylglucoside (M'Batchi et al., 1985). Yet, it is able to transport sucrose, maltose and $\alpha$-phenylglucoside, but not raffinose, probably because of steric hindrance. Sorbitol and stachyose are not transported by the sucrose carrier (M'Batchi and Delrot,
1988) and their presence in the phloem sap, as well as that of raffinose, must be explained by a transport mediated by another carrier, by metabolism inside the conducting complex or by symplastic transport from the mesophyll. The use of the non-permeant sulfhydryl reagent $p$-chloromercuribenzenesulfonic acid (PCMBS) has demonstrated the presence of a thiol protected by the substrate in the active site of the sucrose carrier of broadbean leaf tissue. This property has been used to label differentially the plasmalemma proteins protected by sucrose. The data obtained with purified plasmalemma from sugar beet and from broadbean leaves indicate that an intrinsic polypeptide of $42 \mathrm{kDa}$ is differentially labeled by $N$ ethylmaleimide in the presence of sucrose and not in the presence of the non-transported sucrose analogue palatinose (Pichelin-Poitevin et al., 1987; Gallet et al., 1989). A polyclonal antiserum raised against the $42 \mathrm{kDa}$ polypeptide is able to inhibit selectively uptake of sucrose by leaf protoplasts, but has no effect on the uptake of amino acids and hexoses (Lemoine et al., 1989). These data suggest that the intrinsic $42 \mathrm{kDa}$ polypeptide of the plasmalemma is (part of) the sucrose carrier.

\section{Symplastic loading}

Madore et al. (1986) and Van Bel (1987) have argued that some observations make feasible the possibility that loading into the sieve tubes may be symplastic i.e., via the plasmodesmata. First, in some species, electron microscopy shows more or less numerous plasmodesmata connecting the conducting complex with the surrounding cells (Van Bel, 1987). In addition, several authors have reported on particular cells (paraveinal messophyll), which seem to be located in a strategic position which would allow them to act as cells collecting the 
assimilates from the mesophyll and giving them back to the conducting cells. The leaf of Populus deltoides, studied by Russin and Evert $(1984 ; 1985 a, b)$ provides an excellent example of this situation (Fig. 1). This species possesses a paraveinal mesophyll and there are numerous plasmodesmata between all cell types, including the cells of the conducting complex. In the mesophyll, the highest frequency of plasmodesmata is found between the cells of paraveinal mesophyll and the other cell types. The density of plasmodesmata increases from the mesophyll to the sieve tube and this situation is opposite to that found in broadbean, for example. In soybean, these 'collecting' cells seem to have a more acidic cell wall than the surrounding cells, suggesting that they possess strongly active proton extruding systems (Canny, 1987). Plasmolytic studies with cottonwood also pointed to a situation completely different from that found in the case of apoplastic loading (sugar beet). Indeed, in Populus deltoides, the highest osmotic pressure is not found in the sieve tube, but in the paraveinal mesophyll; there is an osmotic gradient along the palisade cell-bundle sheath cell-companion cell (or vascular

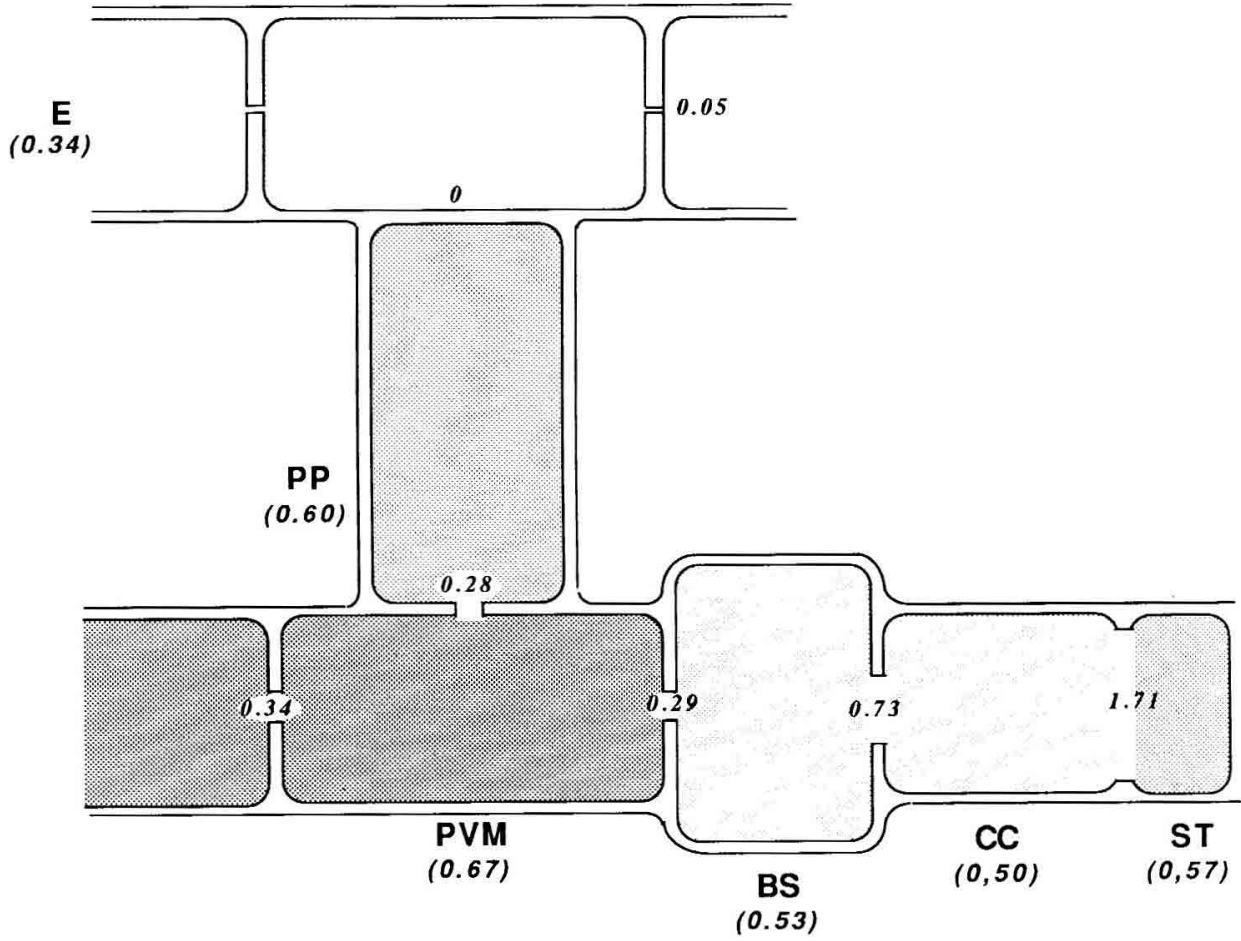

Fig. 1. Osmotic potentials and plasmodesmata frequencies in different cell types of a cottonwood leaf (drawn from the data of Russin and Evert, 1985b). BS: bundle sheath cell; CC: companion cell; E: epidermis; PP: palisade parenchyma cell; PVM: paraveinal mesophyll cell; ST: sieve tube. The osmotic potential (expressed in moles) of each cell in reported between parentheses below the cell name, and the dot density of the intracellular compartment is roughly proportional to this concentration. Numbers in the cell walls refer to the plasmodesmatal frequencies (plasmodesmata per micron interface). 
parenchyma cell) route and along the paraveinal mesophyll-bundle sheath cell-companion cell path. Yet, within the conducting bundle, the osmotic pressure is higher in the sieve tube than in the other cells (companion cell, vascular parenchyma cells). The problem is to know whether these osmotic gradients are due to mobile sugars or to other solutes (ions).

Several structural, ultrastructural and physiological observations therefore suggest that symplastic transport in the leaf may be followed by symplastic loading in some species. The next questions can then be summarized as follows: are the plasmodesmata around the conducting complex open, and if they are open, are they able to build up, or to maintain osmotic gratients? and may these gradients be selective for one mobile form of sugar (sucrose, raffinose, sorbitol, etc.)?

Although this kind of experiment has not yet been conducted with woody species, to our knowledge, injection of fluorescent dyes into the mesophyll cells has shown in several herbaceous species that the dye actually entered the veins but gave no clear demonstration of dye entry into the companion cell-sieve tube complex itself. The data presented above shows that osmotic gradients may be found between cells connected by plasmodesmata.

Now, considering the structure of plasmodesmata (Fig. 2), how can we explain that they would accumulate sucrose in the conducting complex and not hexoses? The diameter of the plasmodesmata is about $50 \mathrm{~nm}$ and the continuity of the plasma membrane from cell to cell is quite evident. A central structure, the desmotubule passes axially along the cylinder. The desmotubule is seen as an extension of the endoplasmic reticulum, but it is not known whether the desmotubule is open or not. The only way to build up a selective concentration gradient across this structure is to hypothesize that the sphinc-

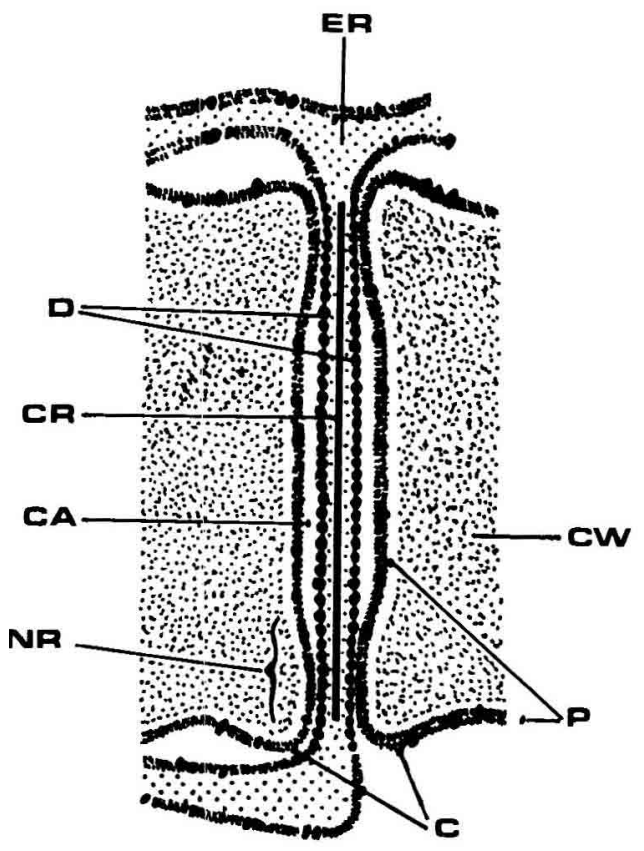

Fig. 2. Schematic representation of a plasmodesma. C: constriction; C:A: central annulus; CR: central rod; CW: cell wall; D: desmotubule; ER: endoplasmic reticulum; NR: neck region; P: plasmalemma (modified from Robards, 1976).

ter and the cytoplasmic annulus would function as a 'one-way' valve or that the desmotubule is open and that active loading is mediated by an energized carrier located on the endoplasmic reticulum or the tonoplast (which communicates with the reticulum). Much additional work is needed to test these hypotheses.

Gamalei and Pakhomova (1980) and Gamalei (1984) surveyed the structure and the repartition of plasmodesmata at the boundary of the conducting complex. According the Gamalei (1984), the structure of the minor veins may be classified into 3 categories (Fig. 3). The type l-vein, characterized by plasmodesmata fields, is typical for plants transporting oligosaccharides (mainly raffinose) and is an adapta- 
tion to symplastic transport (Fig. 3B). Types II (Fig. 3A) and III (Fig. 3C), typical for sucrose transporting species, allow apoplastic transport. Both types I and III, found more frequently in the recent groups of phanerogams, would be derived from type II, found in the older groups of phanerogams. Type I includes gymnosperms and dicotyledon families containing tree species, while types II and III include mainly herbaceous dicotyledons (except Fagaceae, type II).

\section{Possible regulation of loading}

Apart from the numerous metabolic processes which affect the availability of the sugar export pool and which will not be considered here, 2 main factors may affect phloem loading: the cell turgor and hormonal status. Phloem loading is promoted by hyperosmotic media in various species (sugar beet, bean, broadbean, celery), and comparison of the effects of non-permeant and permeant osmotic buffers shows that the important factor is cell turgor. The effects of cell turgor on loading may be due in part to the sensitivity of the transmembrane potential difference to the osmotic conditions (Li and Delrot, 1987). Yet the effects of turgor on the plasma membrane ATPase are not sufficient to explain the osmotic sensitivity of loading and other phenomena must be involved. Furthermore, due to the large osmotic changes needed to affect loading in vitro, it is not known what part osmotic regulation of this process actually plays in vivo.

Various reports have concluded that phytohormones could directly control phloem loading. Malek and Baker (1978) found that auxin promoted phloem loading in castor bean, while Vreugdenhil (1983) reported inhibition of sucrose uptake by abscisic acid in discs prepared from the cotyledons of the same species. More recently, Daie (1987) studied the effects of

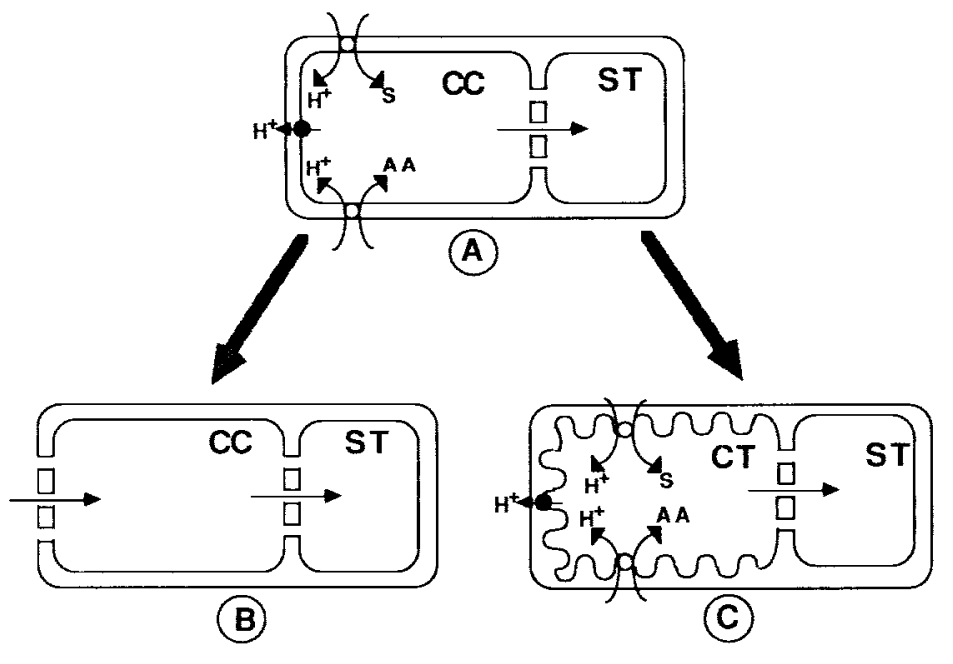

Fig. 3. Different types of phloem-conducting complexes. A: type II; B: type I; C: type III. According to Gamalei (1984), types I and III would derive from type II (bold arrows). The proton-pump ATPase (O), sucrose (S) and amino acid (AA) cotransports are shown. Horizontal arrows indicate the main direction of assimilate flux. CC: companion cell; ST: sieve tube; CT: transfer cell. 
gibberellic acid and auxin on phloem loading in isolated vascular bundles and phloem tissue of celery. She found that both hormones $(1 \mu \mathrm{M})$ were able to stimulate sucrose uptake in these materials within $2 \mathrm{~h}$ of treatment. This effect was also apparent on the uptake of mannitol, which is also translocated in celery, but could not be detected with 3-O-methyglucose, which does not enter the veins. The hormonal effects were therefore attributed to phloem loading. Again, the mechanism of this regulation and the actual part it plays in vivo remain to be elucidated.

Phloem loading and carbon partitioning can be affected in the short-term by artificial manipulation of the source-sink relationships. For example, in broadbean, heat-girdling of a petiole still attached to the plant leads to an apparent inhibition of loading (Ntsika and Delrot, 1986), which seems to be due to the diversion of ${ }^{14} \mathrm{C}$ from the mobile pool to starch (Grusak, Delrot and Ntsika, unpublished data).

\section{Phloem unloading and accumulation by the receiving cells}

While the pathway for loading may depend upon the species investigated, the pathway for phloem unloading depends mainly upon the receiving organs, not only on the species.

In young importing leaves or in root tips, ultrastructural data and various other approaches (use of impermeant inhibitors) indicate that unloading is symplastic (Fig. 4A). In this case, the rate of import is directly dependent upon the metabolic activity of the tissue, which will consume the imported assimilates.

In the stems of various herbaceous species (sugar cane, broadbean, bean), un- loading is apoplastic. Using broadbean stem segments, Aloni et al. (1986) showed that sucrose efflux from the phloem was mediated by a carrier sensitive to PCMBS. Indeed, the efflux of preloaded $\left[{ }^{14} \mathrm{C}\right]$ sucrose was enhanced when unlabeled sucrose was present in the efflux medium, compared to a control. This exchange mechanism is inhibited by PCMBS. This efflux is not active because it is stimulated by the addition of protonophores. After efflux from the phloem into the apoplast of the stem, sucrose is either hydrolyzed by a cell wall invertase, as in sugar cane (Fig. $4 \mathrm{~B})$, or not hydrolyzed as in broadbean (Fig. 4C). The resulting sugars, either hexoses or sucrose, are then actively taken up by the receiving cells.

In the stems of trees (Populus), the density of plasmodesmata $\left(8 / \mu \mathrm{m}^{2}\right)$ in the ray cells is almost as high as in the paraveinal cells of the leaf and allows radial transport of sugars via the symplastic pathway (Sauter and Kloth, 1986).

In fruits, the examples studied so far indicate that the first steps of unloading in the maternal tissues are symplastic but there is a symplastic discontinuity between the 2 generations and uptake of assimilates by the embryo occurs necessarily from the apoplast. In this case, the limiting step for import is the rate of uptake across the plasmalemma of the embryo cells, which in turn clepend upon the metabolism and the compartmentation of assimilate in the receiving cell. Two examples illustrate this configuration. The first one is the fruit of bean, investigated by Thorne (1985). In this material, unloading from the conducting complex in the seed coat (i.e., unloading sensu stricto) is symplastic and then the assimilates are also released into the apoplast at the interface between the 2 generations (Fig. 4D). Sucrose is not split before being absorbed by the cotyledons. In the fruit of rnaize, investigated by Shannon et al. (1986), unloading from the sieve 

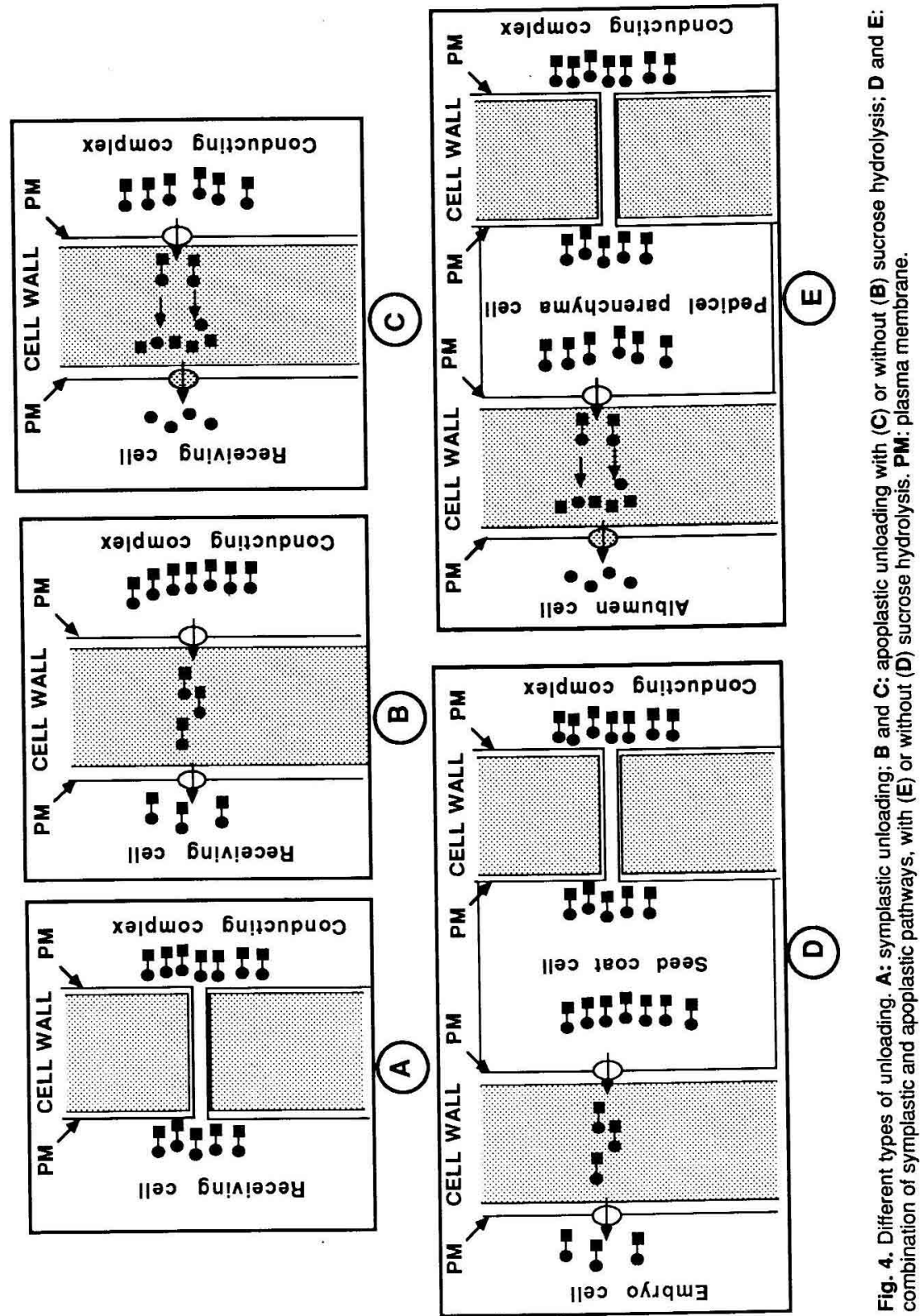
element-companion cell complex is also symplastic (Fig. 4E). Assimilates then apparently enter the apoplast of the placenta-chalaza. However, in contrast to the case described above, they are hydrolyzed in the apoplastic compartment. Indeed, hexoses constitute over $80 \%$ of the carbohydrate released into the apoplast interface between the 2 generations. Assimilates are then taken up by the albumen, presumably as hexoses, and this is facilitated by the conversion of the outer layer of albumen into transfer cells, which are characterized by extensive wall ingrowths. It must be stressed that sucrose hydrolysis, even when it occurs, may not be a necessary prerequisite for sugar accumulation by the sink cells, as has been demonstrated for the taproot of sugar beet (Lemoine et al., 1988). Inversion of sucrose by a cell wall invertase prevents its retrieval by the conducting complex (Eschrich, 1980) and it increases the osmotic pressure in that cell wall.

Possible regulation of unloading may be osmotic or hormonal, as for loading. For example, Aloni et al. (1986) have shown that unloading of assimilates from the stem of broadbean was decreased when the mannitol concentration of the medium was changed from 0 to $400 \mathrm{mM}$ mannitol, but opposite results have been reported with legume fruits (Wolswinkel, 1985). Studies made with different sink organs agree that high solute concentration in the apoplast promotes assimilate uptake into the receiving cells (Wolswinkel, 1985).

Concerning hormonal control, Saftner and Wyse (1984) showed that treatment by abscisic acid enhanced the active component of sucrose uptake in sugar beet root discs, while auxin decreased this uptake 2-fold. These effects, clearly visible within 30 min of treatment, were optimal at $1-10 \mu \mathrm{M}$ for both hormones. $\mathrm{K}^{+}$or auxin prevented the response to abscisic acid but cytokinins and gibberellic acid did not.
These data show that storage in receiving cells may be regulated by hormones. As regards unloading from the phloem, sensu stricto, Clifford, et al. (1986) have reported that import of $\left[{ }^{14} \mathrm{C}\right]$ assimilates in bean pods was promoted by benzylaminopurine and abscisic acid. However, this stimulation was rather weak and did not last for a long time. As in the case of loading, the hormonal effects on unloading are still poorly understood.

In summary, long distance transport and, therefore, the growth of the plant are dependent upon membrane activities at the source and the sink levels, but we still know little about the details of some of these activities, especially in trees. It is clear that various strategies have been developed in the plant kingdom (apoplastic or symplastic loading, apoplastic or symplastic urloading, chemical continuity or non-continuity of the transported substrates) to ensure the transport and the compartmentation of nutrients in the plant.

\section{References}

Aloni B., Wyse R.E. \& Griffith S. (1986) Sucrose transport and phloem unloading in stem of Vicia faba: possible involvement of a sucrose carrier and osmotic regulation. Plant Physiol. 81, 482-486

Canny M.J. (1987) Locating active proton extrusion pumps in leaves. Plant Cell Environ. 10, 271-274

Cliford P.E., Offler C. \& Patrick J.W. (1986) Growth regulators have rapid effects on photosynthate unloading from seed coats of Phaseo lus vulgaris L. Plant Physiol. 80, 635-637

Daie J. (1987) Interaction of cell turgor and hormones on sucrose uptake in isolated phloem of celery. Plant Physiol. 84, 1033-1037

Delrot S. (1987') Phloem loading: apoplastic or symplastic? Plant Physiol. Biochem. 25, 667676

Delrot S. (1989) Loading of photoassimilates. In: Transport of Assimilates. (Baker D.A. \& Mil- 
burn J.A., eds.), Longman Scientific, London, pp. $166-205$

Delrot S. \& Bonnemain J.L. (1985) Mechanism and control of phloem transport. Physiol. Vég. 23, $199-220$

Eschrich W. (1980) Free space invertase, its possible role in phloem unloading. Ber. Dtsch. Bot. Ges. 93, 363-378

Faucher M., Bonnemain J.L. \& Doffin M. (1982) Effets de refroidissements localisés sur la circulation libérienne chez quelques espèces avec ou sans protéines $P$ et influence du mode de refroidissement. Physiol. Vég. 20, 395-405

Gallet O., Lemoine R., Larsson C. \& Delrot S. (1989) The sucrose carrier of the plant plasma membrane. I. Differential affinity labeling. Biochim. Biophys. Acta 978, 56-64

Gamalei Y.V. (1984) Structure of leaf minor veins and forms of sugar transport. Sov. Plant Physiol. 277, 1513-1516

Gamalei Y.V. \& Pakhomova M.V. (1980) DIstribution of plasmodesmata and parenchyma transport of assimilates in the leaves of several dicots. Sov. Plant Physiol. 28, 901-912

Giaquinta R.T. (1983) Phloem loading of sucrose. Annu. Rev. Plant Physiol. 34, 347-387

Hocking P.J. (1980) The composition of the phloem exudate and xylem sap from tree tobacco (Nicotiana glauca). Ann. Bot. 45, 633-640

Kursanov A.L., Kulikova A.L. \& Turkina M.V. (1983) Actin-like protein from the phloem of Heracleum sosnowsky. Physiol. Vég. 21, 353359

Lemoine R., Daie J. \& Wyse R. (1988) Evidence for the presence of a sucrose carrier in immature sugar beet taproots. Plant Physiol. $86,575-580$

Lemoine R., Delrot S., Gallet O. \& Larsson C. (1989) The sucrose carrier of the plant plasma membrane. II. Immunological evidence. Biochim. Biophys. Acta 978, 65-71

Li Z.S. \& Delrot S. (1987) Osmotic dependence of the transmembrane potential difference of broadbean mesocarp cells. Plant Physiol. 84, 895-899

Madore M.A., Oross J.W. \& Lucas J.W. (1986) Symplastic connections in lpomoea tricolor source leaves. Demonstration of functional symplastic connections from mesophyll to minor veins by a novel dye-tracer method. Plant Physiol. 82, 432-442
Malek F. \& Baker D.A. (1978) Effects of fusicoccin on proton cotransport of sugars in the phloem of willow Planta 90, 230-235

M'Batchi B. \& Delrot S. (1988) Stimulation of sugar exit from leaf tissues of Vicia faba $L$. Planta 174, 340-348

M'Batchi B., Pichelin D. \& Delrot S. (1985) The effect of sugars on the binding of $\left[{ }^{203} \mathrm{Hg}\right]-p$-chloromercuribenzenesulfonic acid to leaf tissues. Plant Physiol. 79, 537-542

Ntsika G. \& Delrot S. (1986) Changes in apoplastic and intracellular leaf sugars induced by the blocking of export in Vicia faba. Physiol. Plant. 68, 145-153

Pichelin-Poitevin D., Delrot S., M'Batchi B. \& Everat-Bourbouloux A. (1987) Differential labeling of membrane proteins by Nethylmaleimide in the presence of sucrose. Plant Physiol. Biochem. 25, 597-607

Robards A.W. (1976) Plasmodesmata in higher plants. In: Intercellular Communications in Plants: Studies on Plasmodesmata. (Gunning B.E.S. \& Robards A.W., eds.), Springer-Verlag. Berlin, pp. 1-57

Russin W.A. \& Evert R.F. (1984) Studies on the leaf of Populus deltoides (Salicaceae): morphology and anatomy. Am. J. Bot. 71, 1398-1415

Russin W.A. \& Evert R.F. (1985a) Studies on the leaf of Populus deltoides (Salicaceae): quantitative aspects, and solution concentrations of the sieve-tube members. Am. J. Bot. $72,487-500$

Russin W.A. \& Evert R.F. (1985b) Studies on the leaf of Populus deltoides (Salicaceae): ultrastructure, plasmodesmatal frequency, and solute concentrations. Am. J. Bot. 72, 12321247

Saftner R.A. \& Wyse R.E. (1984) Effect of plant hormones on sucrose uptake by sugar beet root tissue discs. Plant Physiol. 74, 951-955

Sauter J.J. \& Kloth S. (1986) Plasmodesmatal frequency and radial translocation rates in ray cells of poplar (Populus $x$ canadensis Moench 'robusta'). Planta 168, 377-380

Shannon J.C., Porter G.A. \& Knievel D.P. (1986) Phloem unloading and transfer of sugars into developing corn endosperm. In: Phloem Transport. (Cronshaw J., Lucas W.J. \& Giaquinta R.T., eds.), Alan Liss, New York, pp. 265-277

Thorne J.H. (1985) Phloem unloading of $C$ and $\mathrm{N}$ assimilates in developing seeds. Annu. Rev. Plant Physiol. 36, 317-343 
Turkina M.V., Kulikova A.L., Sokolov O.I., Bogatyrev V.A. \& Kursanov A.L. (1987) Actin and myosin filaments form the conducting tissues of Heracleum sosnowskyi. Plant Physiol. Biochem. 25, 689-696

Van Bel A.J.E. (1987) The apoplast concept of phloem loading has no universal validity. Plant Physiol. Biochem. 25, 677-686

Vreugdenhil D. (1983) Abscisic acid inhibits phloem loading of sucrose. Physiol. Plant. 57, 463-467
Vreugdenhil D. (1985) Source-to-sink gradient of potassium in the phloem. Planta 163, 238240

Wolswinkel P. (1985) Phloem unloading and turgor-sensitive transport: factors involved in sink control of assimilate partitioning. Physiol. Plant. 65, 331-339

Ziegler H. (1975) Nature of transported substances. In: Encyclopedia of Plant Physiology, 1, Transport in Plants, I. Phloem Transport (Zimmermann M.H. \& Milburn J.A., eds.), Springer-Verlag, Berlin, pp. 395-431 